\title{
Dependence on a Retinophilin/Myosin Complex for Stability of PKC and INAD and Termination of Phototransduction
}

\author{
Kartik Venkatachalam, ${ }^{1 *}$ David Wasserman, ${ }^{1 *}$ Xiaoyue Wang, ${ }^{1}$ Ruoxia Li, ${ }^{1}$ Eric Mills, ${ }^{1}$ Rebecca Elsaesser, ${ }^{1}$ \\ Hong-Sheng $\mathrm{Li}^{2}{ }^{2}$ and Craig Montell ${ }^{1}$ \\ ${ }^{1}$ Departments of Biological Chemistry and Neuroscience, Center for Sensory Biology, The Johns Hopkins University School of Medicine, Baltimore, \\ Maryland 21205, and 2Department of Neurobiology, University of Massachusetts Medical School, Worcester, Massachusetts 01605
}

\begin{abstract}
Normal termination of signaling is essential to reset signaling cascades, especially those such as phototransduction that are turned on and off with great rapidity. Genetic approaches in Drosophila led to the identification of several proteins required for termination, including protein kinase C (PKC), NINAC (neither inactivation nor afterpotential C) p174, which consists of fused protein kinase and myosin domains, and a PDZ (postsynaptic density-95/Discs Large/zona occludens-1) scaffold protein, INAD (inactivation no afterpotential D). Here, we describe a mutation affecting a poorly characterized but evolutionarily conserved protein, Retinophilin (Retin), which is expressed primarily in the phototransducing compartment of photoreceptor cells, the rhabdomeres. Retin and NINAC formed a complex and were mutually dependent on each other for expression. Loss of retin resulted in an age-dependent impairment in termination of phototransduction. Mutations that affect termination of the photoresponse typically lead to a reduction in levels of the major rhodopsin (Rh1) to attenuate signaling. Consistent with the slower termination in retin ${ }^{1}$, the mutant photoreceptor cells exhibited increased endocytosis of Rh1 and a decline in Rh1 protein. The slower termination in retin ${ }^{1}$ was a consequence of a cascade of defects, which began with the reduction in NINAC p174 levels. The diminished p174 concentration caused a decrease in INAD. Because PKC requires interaction with INAD for protein stability, this leads to reduction in PKC levels. The decline in PKC was age dependent and paralleled the onset of the termination phenotype in retin $^{l}$ mutant flies. We conclude that the slower termination of the photoresponse in retin ${ }^{1}$ resulted from a requirement for the Retin/NINAC complex for stability of INAD and PKC.
\end{abstract}

\section{Introduction}

Drosophila visual transduction is a genetically tractable model to identify and characterize the roles of proteins involved in sensory signaling (Wang and Montell, 2007). As is the case in mammalian rods and cones, phototransduction in fly photoreceptor cells is initiated by rhodopsin, which engages a heterotrimeric G-protein. In flies, the effector for the G-protein is a phospholipase C (PLC), and the cascade culminates with opening of the TRP (Transient Receptor Potential) and TRPL (Transient Receptor Potential-Like) cation channels. This contrasts with rod and cone phototransduction, which concludes with closure of cGMP-gated channels (Fu and Yau, 2007). However, a third class of mammalian photoreceptor cells with non-image-forming functions, the intrinsically sensitive retinal ganglion cells, may function through a cascade akin to fly phototransduction (Hankins et al., 2008).

\footnotetext{
Received May 27, 2010; accepted July 5, 2010.

This work was supported by National Eye Institute Grant EY08117 (C.M.). We thank the Bloomington Stock Center and the Vienna Drosophila RNAi Center for fly stocks, FlyBase (http://flybase.org/) for essential gene data, Michael Delannoy for preparing EM sections and assistance with EM imaging, Ying Jin, Erin Black, and Daria Nikolaeva for technical assistance, the Microscopy Facility at the Cell Biology Department of The Johns Hopkins University School of Medicine for the use of equipment, and Dr. Jeffrey Corden for use of the LICOR Odyssey infrared imaging system. The authors declare no conflict of interest.

*K.V. and D.W. contributed equally to this work.

Correspondence should be addressed to Craig Montell, Department of Biological Chemistry, The Johns Hopkins University School of Medicine, 855 N. Wolfe Street, Baltimore, MD 21205. E-mail: cmontell@jhmi.edu.

DOI:10.1523/JNEUROSCI.2709-10.2010

Copyright $\odot 2010$ the authors $\quad 0270-6474 / 10 / 3011337-09 \$ 15.00 / 0$
}

Several crucial signaling proteins emerged from genetic studies of Drosophila phototransduction. These include the Drosophila TRP channel, which is essential for activation (Montell and Rubin, 1989; Hardie and Minke, 1992). Another example is NINAC (neither inactivation nor afterpotential C), which consists of linked protein kinase and myosin domains (Montell and Rubin, 1988). NINAC is expressed as two proteins, p132 and p174. The p132 isoform is spatially restricted to cell bodies, whereas p174 is detected exclusively in the microvillar portion of the photoreceptor cells, the rhabdomeres (Porter et al., 1992), which is the phototransducing compartment (Wang and Montell, 2007). Mammalian proteins comprising fused protein kinase and myosin domains (myosin III) are expressed in photoreceptor cells and in the inner ear, and mutations in human myosin III underlie one type of non-syndromic hearing loss (Dosé and Burnside, 2000; Walsh et al., 2002). NINAC binds to calmodulin (Porter et al., 1993, 1995), and disruption of NINAC p174 but not p132 impairs rapid termination and causes an age-dependent electrophysiological phenotype that suggests that there is a decline in rhodopsin concentration (Porter et al., 1992). Moreover, NINAC p174 has been suggested to accelerate the binding of arrestin to lightactivated rhodopsin, through a $\mathrm{Ca}^{2+} /$ calmodulin-dependent mechanism (Liu et al., 2008).

Rapid termination of phototransduction is essential to reset the photoreceptor cells so they can respond appropriately to subsequent light stimulation. In addition to NINAC p174, other proteins required for normal termination include arrestin (Dolph et al., 
1993), calmodulin (Porter et al., 1993; Scott et al., 1997), an eye-enriched protein kinase C (PKC) (Smith et al., 1991), RDGC (retinal degeneration C, isoform D) (Vinós et al., 1997; Lee and Montell, 2001), PLC (Bloomquist et al., 1988), which also acts as a GTPase activating protein (Cook et al., 2000; T. Wang et al., 2008), and the PDZ (postsynaptic density-95/Discs Large/zona occludens1)-containing scaffold protein INAD (inactivation no afterpotential D) (Pak, 1979; Shieh and Niemeyer, 1995; Popescu et al., 2006). INAD binds to multiple proteins required for the photoresponse, several of which, including TRP, PLC, and PKC, depend on interactions with INAD for stable expression in the rhabdomeres (Huber et al., 1996; Chevesich et al., 1997; Tsunoda et al., 1997; Xu et al., 1998). The concentrations of other INAD binding proteins, such as NINAC p174, are not affected by associations with this scaffold protein (Wes et al., 1999).

Here, we used ends-out homologous recombination to knock-out a gene, retinophilin (retin; also referred to as undertaker) (Mecklenburg, 2007; Cuttell et al., 2008), which was expressed predominantly in the eye. The retin gene encodes a poorly characterized protein conserved from flies to humans. We found that retin mutant flies displayed age-dependent impairment in termination of the photoresponse. The appearance of this phenotype coincided with an age-dependent decrease in the concentration of PKC because of a series of defects in the retin mutant. Specifically, we found that Retin formed a complex with NINAC p174, and loss of Retin caused instability of 174, which in turn resulted in a decrease in the concentration of INAD. Because $\mathrm{PKC}$ requires interaction with INAD for stability, the level of PKC fell in retin mutant flies. Thus, the Retin/NINAC complex was required for stability of INAD and PKC and normal termination of the photoresponse.

\section{Materials and Methods}

Fly stocks. Upstream activating sequence (UAS)-retin-RNA interference (RNAi) was obtained from Vienna Drosophila RNAi center, and $D f(3 R) E D 5147$ was from the Bloomington Stock Center. Other fly lines were described previously.

Generation of retin ${ }^{1}$. We used ends-out homologous recombination (Gong and Golic, 2003) to create a mutation in retin (National Center for Biotechnology Information accession number NM_141263.2; also referred to as undertaker) (Cuttell et al., 2008). One fragment ( -3000 to -2 ) was inserted between the NotI and SphI sites of pw35. A second fragment (3000 nucleotides $3^{\prime}$ to the nucleotide at position +89 ) was inserted into the BamHI site. Male transformant flies containing the transgene on the second chromosome were mated to female $y w$; $\mathrm{P}[70 \mathrm{FLP}] 11 \mathrm{P}[70 \mathrm{I}-\mathrm{SceI}] 2 \mathrm{~B}$ noc ${ }^{\text {Sco }} / \mathrm{CyO}$ flies. Progeny were heat shocked $\left(37^{\circ} \mathrm{C}\right)$ for $1 \mathrm{~h}$, and $\sim 10 \mathrm{~d}$ later females were crossed to $w^{1118}$ males. Flies
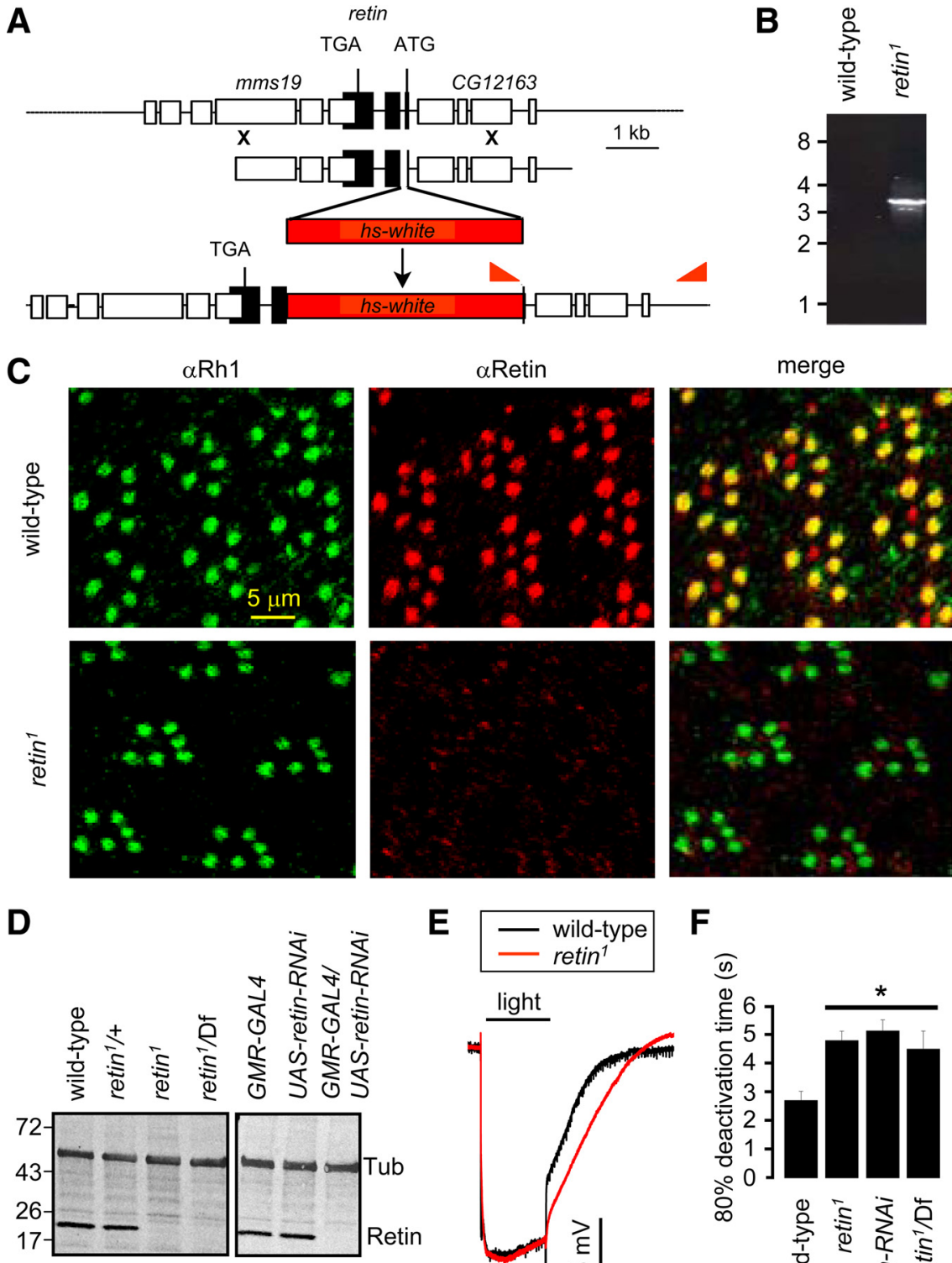

E
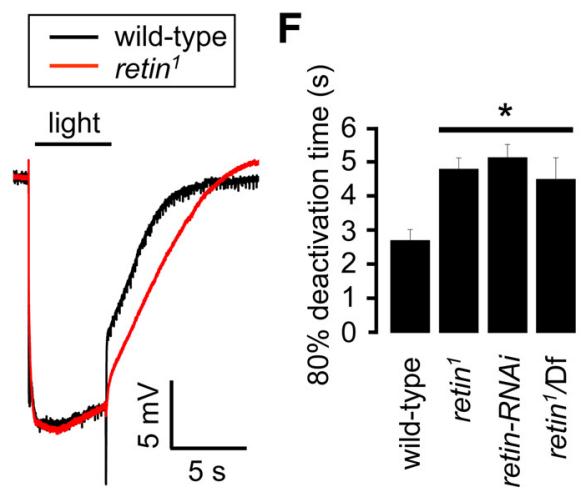

Figure 1. Loss of the Retin leads to delayed termination. $A$, The retin genomic loci in wild type and retin ${ }^{1}$. The genes flanking retin, $m$ ms 19, and CG12163 are indicated. The red triangles indicate the primers used for the PCR analyses in $\boldsymbol{B}$. B, PCR confirmation xpression patterns of Rh1 (green) and Retin (red) in retinal cross-sections. D, Western blots of head extracts probed with Retin and Tubulin (Tub) antibodies. Protein size markers are indicated in kilodaltons. $\boldsymbol{E}$, ERGs performed in dark-adapted 9-d-old wild-type and retin ${ }^{7}$ flies. $\boldsymbol{F}$, Quantification of the $80 \%$ deactivation times. Error bars indicate the means \pm SEM. ${ }^{*} p<0.05$, ANOVA, $n \geq 3$.

with $w^{+}$transgenes that moved to the third chromosome were screened by PCR to identify targeting in the retin gene. Knock-out of Retin was confirmed by Western blotting.

Generation of Retin antibodies. A retin cDNA (encoding residues 3-198) was subcloned into pGEX5X-1 (GE Healthcare). The glutathione S-transferase fusion protein was expressed in Escherichia coli BL21 codon-plus (Stratagene), purified using glutathione agarose beads (GE Healthcare), and injected into rabbits (Covance).

Immunolocalizations. Fly heads were hemisected, fixed in paraformaldehyde, and embedded in LR White resin as described previously (Porter and Montell, 1993). Cross-sections $(0.5 \mu \mathrm{m})$ were blocked using $5 \%$ goat serum in PBS for $1 \mathrm{~h}$, incubated for $1 \mathrm{~h}$ with primary antibodies (rabbit anti-Retin at 1:1000 or mouse anti-Rh1 at 1:1000), diluted in blocking buffer, washed with PBS, and incubated for 1 h with Alexa Fluor 488labled goat anti-mouse and/or Alexa Fluor 568-labled goat-anti-rabbit (Invitrogen) diluted at 1:500 in blocking buffer. Cross-sections were 
A

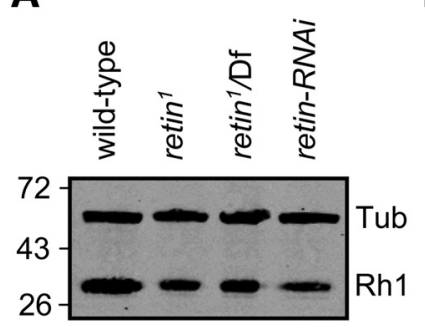

B

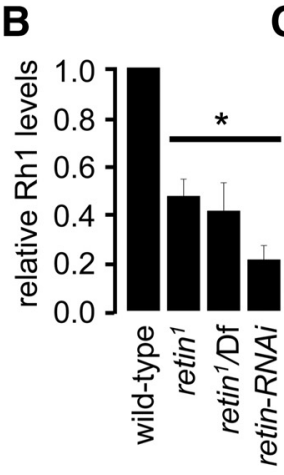

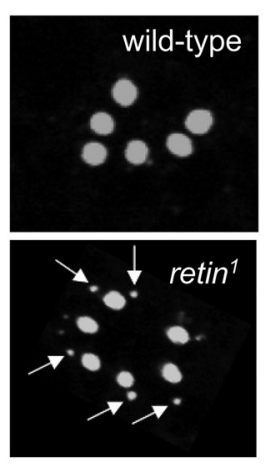

D

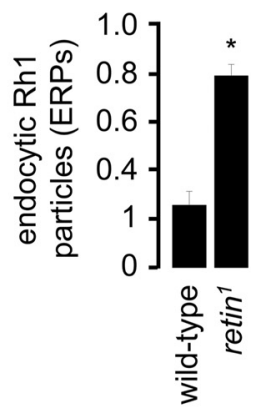

E

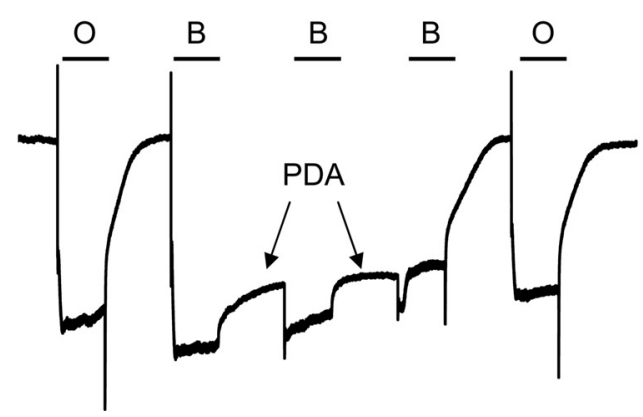

$\mathbf{F}$ retin 1

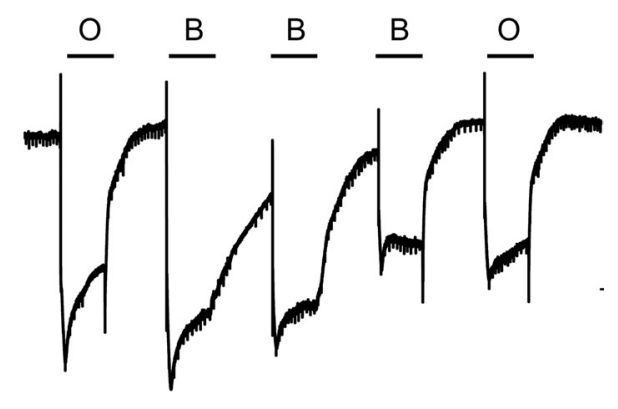

Figure 2. Loss of Rh1 in retin flies. $\boldsymbol{A}$, Western blot containing head extracts from 7-d-old wild-type and retin-deficient flies probed with Rh1 and Tubulin antibodies. Protein size markers are indicated in kilodaltons. $\boldsymbol{B}$, Relative Rh1 levels based on Western blots. Error bars indicate \pm SEM. ${ }^{*} p<0.05$, ANOVA, $n \geq 3$. C, Confocal images showing the appearance of ERPs (arrows) in 7-d-old retin $^{7}$. D, Number of ERPs/rhabdomere. Error bars indicate \pm SEM. ${ }^{*} p<0.05$, Student's $t$ test, $n \geq 3$. E, PDA in 7-d-old wild-type flies. The lines above the ERGs indicate $5 s$ pulses of orange $(0)$ or blue (B) lights. $F$, Loss of PDA in 7-d-old retin?.

washed with PBS and mounted with Vectashield (Vector Laboratories). All incubations were done at room temperature. Images were acquired using a Carl Zeiss 510 Meta Confocal Microscope.

Western blots. Fly heads were homogenized in SDS-sample buffer, and proteins were fractionated by SDS-PAGE and transferred to Hybond-C Extra Nitrocellulose membranes (GE Healthcare) in Tris-glycine buffer. The blots were probed with mouse anti-tubulin (1:1000 dilution; Developmental Studies Hybridoma Bank), rabbit anti-Retin (1:1000 dilution), rabbit anti-Rh1 (1:1000; gift from D. Ready, Purdue University, West Lafayette, IN), rabbit anti-NINAC ( $\alpha$ ZB551) (1:1000 dilution) (Montell and Rubin, 1988), rabbit anti-NORPA (no receptor potential A) (PLC) antibodies (1:1000 dilution) (Wang et al., 2005), rabbit anti-arrestin 2 (Arr2) antibodies (1:500 dilution; S. J. Lee and C. Montell, unpublished data), and rabbit anti-PKC antibodies (1:1000 dilution) (Li and Montell, 2000). The blots were probed with IRDye 680 donkey anti-rabbit IgG (LI-COR) or IRDye 800 donkey anti-mouse IgG (LI-COR) and detected with the Odyssey infrared imaging system (LI-COR).

Electroretinogram recordings. To perform electroretinograms (ERGs), we filled two glass microelectrodes with Ringer's solution, which we inserted into drops of electrode cream placed on the surfaces of the compound eye and the thorax. We stimulated the eyes using a Newport light projector (model 765), and the ERG signals were amplified with a Warner Instruments electrometer IE-210. We recorded the waveforms with a Powerlab 4/30 analog-to-digital converter (AD Instruments) and the Lab Chart version 6.1 program (AD Instruments). We measured prolonged depolarization afterpotential (PDAs) using five pulses of orange (580) or blue (480) light in the following order: orange, blue, blue, orange, orange ( $5 \mathrm{~s}$ pulse separated by $7 \mathrm{~s}$ intervals). All recordings were conducted at room temperature.

Transmission electron microscopy. Heads were dissected from flies reared under a light/dark cycle or in constant darkness, fixed in glutaraldehyde, and embedded in LR White resin as described previously (Porter et al., 1992). Sections ( $85 \mathrm{~nm}$ ) prepared at a depth of $30 \mu \mathrm{m}$ were examined by transmission EM using a Carl Zeiss FEI Tecnai 12 electron microscope. The images were acquired using a Gatan camera (model 794) and Gatan Digital Micrograph software and converted into tiff files.
Optical neutralization technique. To assay the numbers of rhabdomeres/ommatidium, we used the optical neutralization technique (Franceschini et al., 1981). Each data point was based on $\geq 50$ ommatidia per fly ( $n \geq 3$ flies).

Coimmunoprecipitations. Approximately $15 \mathrm{mg}$ of fly heads were homogenized at $4^{\circ} \mathrm{C}$ in PBS, pH 7.4, containing 1\% Triton X-100, $1 \mathrm{~mm}$ DTT, and $1 \times$ protease inhibitor cocktail (Sigma). The homogenates were centrifuged at $16,000 \times \mathrm{g}$ for $30 \mathrm{~min}$ at $4^{\circ} \mathrm{C}$. Supernatants were incubated with primary antibodies for $2 \mathrm{~h}$ at $4^{\circ} \mathrm{C}$. Protein A Sepharose beads were blocked in homogenization buffer plus $1 \%$ BSA for $30 \mathrm{~min}$ at $4^{\circ} \mathrm{C}$. Blocked beads $(50 \mu \mathrm{l})$ were added to the tubes containing the immune complexes and incubated for $1 \mathrm{~h}$ at $4^{\circ} \mathrm{C}$. Beads plus immune complexes were pelleted by low-speed $(3500 \times g)$ centrifugation at $4^{\circ} \mathrm{C}$ and washed three times with homogenization buffer. Immune complexes were eluted with $2 \times$ SDS sample buffer, and proteins were detected by Western blotting.

\section{Results}

\section{Generation of the retin ${ }^{1}$ mutant}

Most genes known to function in phototransduction are expressed predominantly in the eye (Wang and Montell, 2007). Therefore, to identify new candidate genes required for Drosophila phototransduction, we previously conducted a genome-wide screen for genes expressed predominantly in the eye (Xu et al., 2004). A gene referred to as retinophilin (retin) (Mecklenburg, 2007) was among the most eye-enriched genes (146.7-fold) that have not been subjected to functional analysis (Xu et al., 2004). The retin gene (83A1 on the third chromosome) encodes a 198 aa protein with four tandem 23-24 aa MORN (membrane occupation and recognition nexus) domains, originally identified in a group of proteins referred to as junctophilins (Takeshima et al., 2000). Retin is not a junctophilin because it is much smaller than these $\sim 600-1000$ aa proteins, lacks the typical C-terminal transmembrane domain, and does not share sequence homology with junctophilins outside of the MORN domains. Rather, Drosophila 
Retin is a member of a distinct group of poorly characterized proteins that is conserved from flies to humans. Human Retin shares $\sim 50 \%$ identity with the fly Retin over a 140 aa region that includes the four MORN domains and most of the $\mathrm{C}$ terminus.

To characterize the role of retin in photoreceptor cells, we generated the retin ${ }^{1}$ mutation by homologous recombination (Fig. 1A). The deletion removed the initiation codon and the following two methionine codons. The next methionine in the sequence does not occur until residue 132. Evidence that the deletion was targeted to the retin locus was that the predicted $3.1 \mathrm{~kb}$ PCR product was produced in retin ${ }^{1}$ but not wild-type flies, using primers corresponding to the white marker gene and a genomic region outside of the targeting construct (Fig. $1 A, B$ ).

\section{Retin was detected exclusively in the rhabdomeres}

To define the subcellular distribution of Retin, we raised antibodies to the Retin protein. The antibodies appeared to recognize Retin because it stained a protein near the predicted molecular weight of $22.7 \mathrm{kDa}$ in wild type (Fig. $1 \mathrm{D}$ ). This protein was absent in retin ${ }^{1}$ or in flies containing the retin ${ }^{1}$ mutation in trans with a deficiency chromosome (Df) that contains a large deletion covering several genes, including retin (Fig. 1D). Because the Retin protein was absent in retin ${ }^{l}$ and $\operatorname{retin}^{l} / \mathrm{Df}$, we concluded that retin ${ }^{1}$ is a null mutation.

The Drosophila compound eye consists of $\sim 800$ ommatidia, each of which includes eight photoreceptor cells, although only seven are present in any cross-sectional plane. Each photoreceptor cells includes a rhabdomere, which is the functional equivalent of rod and cone outer segments. The major rhodopsin, Rh1, which is expressed in the six outer photoreceptor cells (R1-R6), is a marker for rhabdomere-specific staining. We found that the Retin antibodies stained wild-type rhabdomeres exclusively, because it colocalized with Rh1 in the R1-R6 cells but did not label the extra-rhabdomeral cell bodies or the rhabdomeres of retin $^{1}$ flies (Fig. 1C).

\section{Requirement for retin for termination of the photoresponse}

To determine whether the retin ${ }^{1}$ mutation affected the light response, we performed ERGs, which are extracellular recordings that assay the summed responses of all retinal cells to light. We dark-adapted the flies for $10 \mathrm{~min}$ and then exposed them to a $5 \mathrm{~s}$ pulse of light. Wild-type and retin ${ }^{1}$ flies $(9 \mathrm{~d}$ old $)$ displayed corneal negative receptor potentials of similar amplitudes (Fig. $1 E$ ). After cessation of the light stimulus, the response termination was increased significantly in the retin ${ }^{1}$ flies (Fig. $\left.1 E, F\right)\left(t_{(80)}\right.$; wild-type, $2.7 \pm 0.3 \mathrm{~s}$; $\operatorname{retin}^{1}, 4.8 \pm 0.3 \mathrm{~s}$; ANOVA, $p<0.05, n \geq$ $3)$. To obtain an independent fly line with diminished expression of retin, we expressed a UAS-retin-RNAi transgene in photoreceptor cells under the control of the photoreceptor cell-enriched glass multiple reporter-GAL4 (GMR-GAL4). The retin-RNA $i$ was effective, because we did not detect the protein in these transgenic flies (Fig. $1 D$ ). Termination was similarly slowed in retin-RNAi flies and in $\mathrm{retin}^{1} / \mathrm{Df}($ Fig. $1 F)\left(t_{(80)}\right.$; retin-RNAi, $5.1 \pm 0.4 \mathrm{~s}$; $\operatorname{retin}^{1} / \mathrm{Df}, 4.5 \pm 0.3 \mathrm{~s}$; ANOVA, $p<0.05, n \geq 3$ ). These data support the conclusion that the termination defect was not attributable to a background mutation.

\section{Loss of Rh1 in retin ${ }^{1}$ flies}

During Drosophila phototransduction, an increase in the response termination time leads to elevated endocytosis and degradation of $\mathrm{Rh} 1$, which serves as a negative feedback mechanism (Han et al., 2007). Consequently, a decline in Rh1 can provide a sensitive biochemical readout for the onset of a defect in termination. Therefore, we evaluated whether the concentration of
A

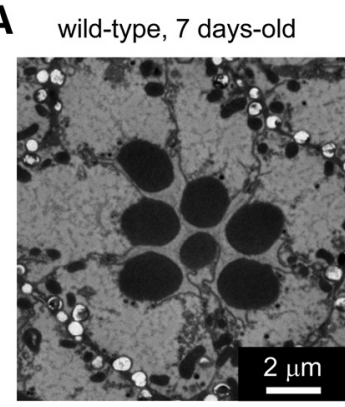

B

retin $^{1}, 7$ days-old

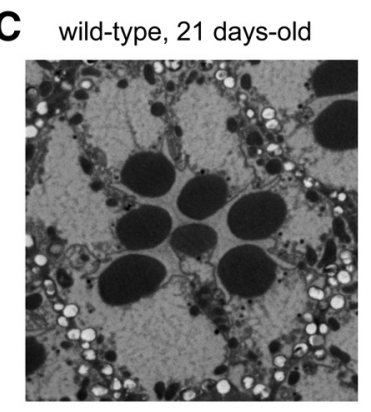

E

retin ${ }^{1}, 21$ days-old dark-reared

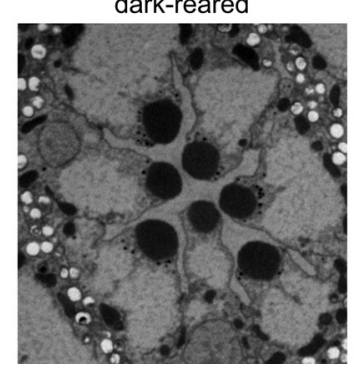

Figure 3. Morphology of retin ${ }^{1}$ photoreceptor cells. $\boldsymbol{A}-\boldsymbol{E}$, Transmission EMs of sections from the distal regions of ommatidia from wild-type and retin ${ }^{1}$ compound eyes. Unless indicated otherwise, the flies were reared under a $12 \mathrm{~h}$ light/dark cycle. $\boldsymbol{A}$, Twenty-one-day-old wild type. $\boldsymbol{B}$, Seven-day-old retin ${ }^{1}$. C, Twenty-one-day-old wild type. $\boldsymbol{D}$, Twenty-one-day-old retin ${ }^{1}$. $\boldsymbol{E}$, Dark-reared 21-d-old retin ${ }^{1}$. F, Average number of rhabdomeres/ommatidium from 15-d-old flies, as quantified using the optical neutralization technique (Franceschini et al., 1981).

Rh1 was decreased in retin ${ }^{1}$. We found that the level of Rh1 was reduced in 7-d-old retin ${ }^{1}$, as well as in $\mathrm{retin}^{1} / \mathrm{Df}$ and retin--RNAi flies (Fig. $2 A, B$ ) (ANOVA, $p<0.05, n \geq 3$ ). This decrease in Rh1 levels correlated with a significant increase in endocytic Rh1 particles (ERPs) in the cell bodies of the retin ${ }^{1}$ photoreceptor cells (Fig. 2C,D) (unpaired Student's $t$ test, $p<0.05, n \geq 3$ ).

We used an additional ERG paradigm to assess whether Rh1 was functionally reduced in retin $^{1}$ flies. In white-eyed flies, transient exposure to blue light results in continuous activation of Rh1. Cessation of this PDA requires exposure to orange light (Fig. $2 E$ ). A decrease in Rh1 levels to $<30 \%$ wild-type levels eliminates the PDA, because the afterpotential depends on an excess of Rh1 over arrestin (Dolph et al., 1993). Consistent with a decrease in $\mathrm{Rh} 1$, the PDA was reduced greatly in 7-d-old retin ${ }^{1}$ flies (Fig. 2F).

The decrease in Rh1 did not appear to be attributable to an age-dependent loss of the rhabdomeres because 7-d-old retin ${ }^{1}$ flies retained the full complement of rhabdomeres, although the size of the rhabdomeres declined slightly (Fig. $3 A, B$ ). Even after $21 \mathrm{~d}$ of a light/dark cycle, the $\mathrm{retin}^{l}$ as well as $\mathrm{retin}^{l} / \mathrm{Df}$ flies retained the complete set of seven rhabdomeres (Fig. $3 C, D, F$ ). Maintaining the flies in the dark did not fully suppress this phenotype, although 
A

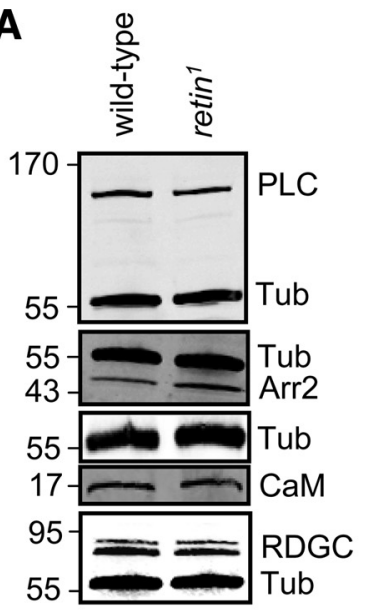

B

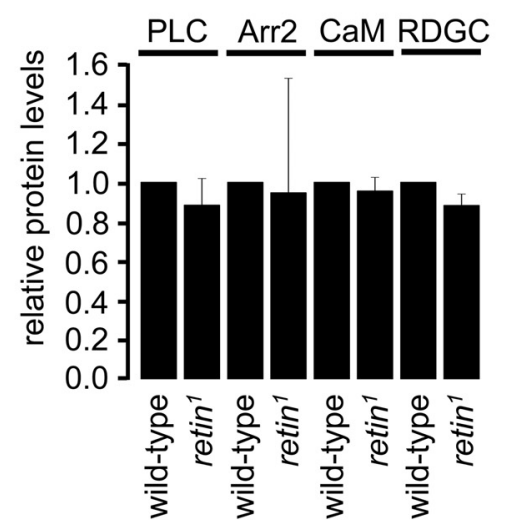

C

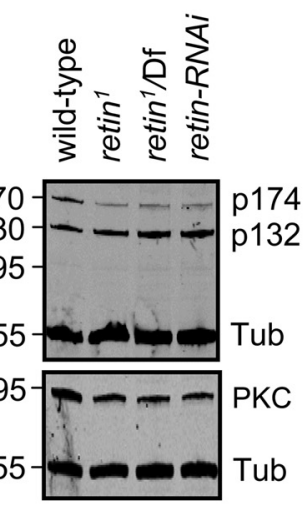

D

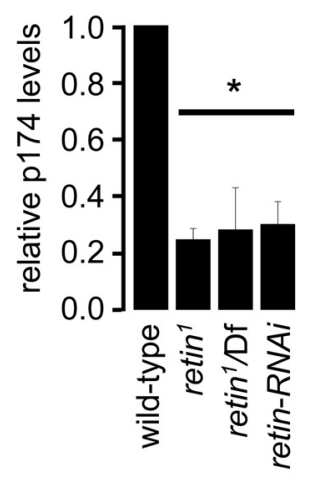

E

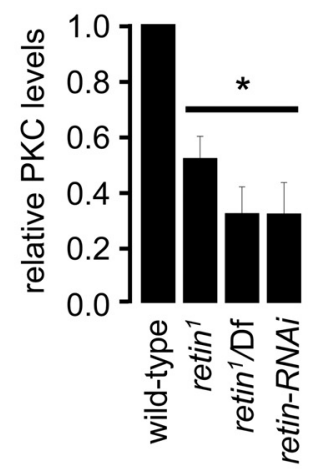

$\mathbf{F}$

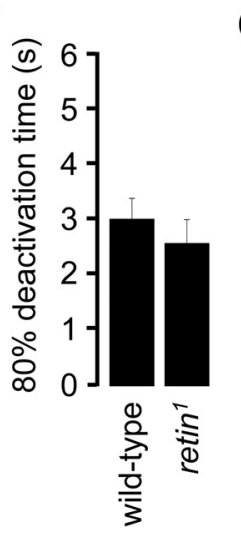

G

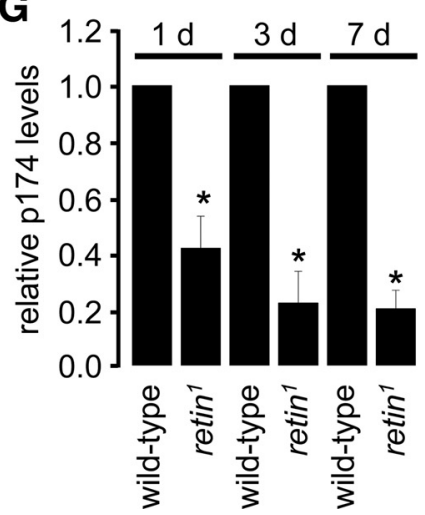

$\mathrm{H}$

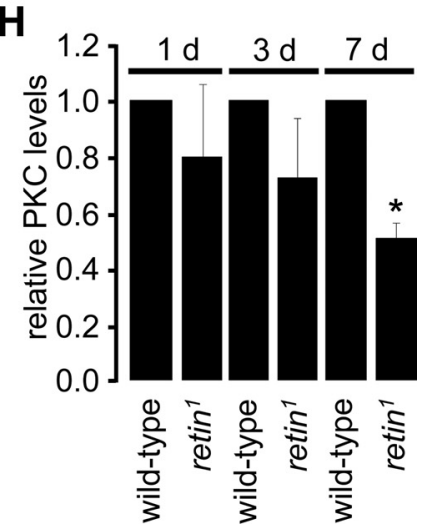

Figure 4. Age-dependent loss of NINAC p174 and PKC in retin ${ }^{7}$. $\boldsymbol{A}$, Western blots using head extracts from 7-d-old flies. Tub, Tubulin; CaM, calmodulin. $\boldsymbol{B}$, Quantification of the relative protein levels from the Western blots. $\boldsymbol{C}$, Western blots showing the levels of NINAC and PKC in head extracts from 7-d-old flies. D, Quantification of the relative p174 levels from the Western blots. $\boldsymbol{E}$, Quantification of the relative PKC levels from the Western blots. $\boldsymbol{F}$, Quantification of the $80 \%$ deactivation times of the light response from 3-d-old flies. G, Quantification of the relative p174 levels from the Western blots from flies of the indicated ages. $\boldsymbol{H}$, Quantification of the relative PKC levels from 1-, 3-, and 7-d-old flies. Error bars indicate \pm SEM. ${ }^{*} p<0.05$, ANOVA, $n \geq 3$.

there was a minor improvement (Fig. 3E). Thus, the decrease in rhabdomere size was not strictly light dependent.

In further support of the correlation between the decline in Rh1 and the onset of slower termination, we found that the concentration of Rh1 in 1- and 3-d-old retin ${ }^{1}$ flies, which display normal termination (supplemental Fig. $1 A$, available at www. jneurosci.org as supplemental material), were not significantly different from wild-type flies (supplemental Fig. $1 B$, available at www.jneurosci.org as supplemental material) $(n \geq 3)$. Furthermore, at $3 \mathrm{~d}$ of age, both wild-type and retin $^{1}$ flies showed normal PDAs (supplemental Fig. $1 C, D$, available at www.jneurosci.org as supplemental material). These results indicated that the delay in the termination of the light response in retin ${ }^{1}$ lead to increased endocytosis and degradation of Rh1.

\section{Protein levels of NINAC and PKC depend on Retin}

Proteins required for rapid termination of the photoresponse include Arr2 (Dolph et al., 1993), the NINAC p174 isoform (Montell and Rubin, 1988; Porter et al., 1992), PKC (encoded by inaC) (Smith et al., 1991), calmodulin (Porter et al., 1993, 1995; Scott et al., 1997), rhodopsin phosphatase (RDGC) (Vinós et al., 1997), and PLC (NORPA) (Bloomquist et al., 1988; Cook et al., 2000; T. Wang et al., 2008). Therefore, we tested whether the levels of any of these proteins were reduced in 7-d-old retin ${ }^{1}$ heads, when the concentration of Rh1 and the PDA had already declined. There were no significant differences in the amounts of PLC, Arr2, calmodulin, or RDGC relative to wild type (Fig. $4 A, B$ ). In contrast, the levels of NINAC p174 and PKC were reduced significantly in $\operatorname{retin}^{1}, \operatorname{retin}^{1} / \mathrm{Df}$, and retinRNAi flies (Fig. 4C-E) (ANOVA, $p<$ $0.05, n \geq 3$ ). However, the concentration of the non-rhabdomeral NINAC isoform, p132, remained unchanged (Fig. 4C) (supplemental Fig. $2 \mathrm{~A}$, available at www. jneurosci.org as supplemental material).

Delay in termination corresponds to an age-dependent decrease in PKC

To address whether the delay in termination in retin $^{1}$ flies arose as a result of a decrease in $\mathrm{p} 174$ or $\mathrm{PKC}$, we examined the time course of loss of p174 and PKC. Although termination was normal in 3 -dold retin ${ }^{1}$ flies (Fig. 4F) (supplemental Fig. $1 A$, available at www.jneurosci.org as supplemental material), the concentration of p174 was already lower than wild type in 1-d-old retin ${ }^{1}$ and decreased further in 3-d-old flies (Fig. 4H) (Student's $t$ test, $p<0.05, n \geq 3)$. Thus, the decline in p174 did not parallel the onset of the termination phenotype. In contrast, the levels of PKC in 1- and 3-d-old retin ${ }^{1}$ flies were similar to those in wild type (Fig. $4 H$ ) (supplemental Fig. 2C, available at www.jneurosci.org as supplemental material) $(n \geq$ $3)$. In 7-d-old retin ${ }^{1}$, the level of PKC were significantly lower than in wild type (Fig. $4 H$ ) (Student's $t$ test, $p<0.05, n=3$ ). Therefore, the onset of the decrease in PKC coincided with the time course of the delay in response termination and the decline in Rh1 levels. Consistent with the conclusion that a twofold reduction in PKC underlay the delay in termination in retin ${ }^{1}$, a similar termination phenotype occurred in inaC $C^{P 209} /+(p k c /+)$ heterozygous flies (N. Wang et al., 2008). As observed in retin ${ }^{1}$ ommatidia, all the photoreceptor cells were still present in 21-d- 
old inaC $C^{P 209} /+$ flies (supplemental Fig. $2 D, E$, available at www.jneurosci.org as supplemental material).

Retin and NINAC p174 form a complex Because the protein concentrations of p174 and PKC decreased in the retin ${ }^{1}$ flies, we tested whether either of the two proteins formed a complex with Retin in vivo. Therefore, we immunoprecipitated Retin from fly heads and probed a Western blot with antibodies that recognized $\mathrm{PKC}$ as well as both forms of NINAC. We found that p174 coimmunoprecipitated with Retin (Fig. 5A). Neither NINAC isoform immunoprecipitated from homogenates prepared from retin ${ }^{1}, \operatorname{retin}^{1} / \mathrm{Df}$, and retinRNAi heads (supplemental Fig. 3B, available at www.jneurosci.org as supplemental material). In a reciprocal experiment, using anti-p174, Retin coimmunoprecipitated with p174 (Fig. 5B). In contrast, Retin did not coimmunoprecipitate with NINAC p132 (Fig. 5B) or PKC (supplemental Fig. $3 A$, available at www.jneurosci.org as supplemental material).

Retin was undetectable in ninaC $C^{\Delta 174}$ Because Retin interacted with p174 and was required for its stability, we addressed whether there was a reciprocal requirement for p174 for expression of Retin. We found that Retin was absent in 7-d-old flies lacking the two NINAC proteins $\left(\right.$ ninaC $\left.{ }^{\mathrm{P} 235}\right)$ or p174 only $\left(\right.$ ninaC $\left.^{\Delta 174}\right)$ (Fig. 5C). Furthermore, we did not detect the Retin protein in nina $C^{\Delta 174}$ flies that were $1 \mathrm{~d}$ old or even in flies that were only $30 \mathrm{~min}$ after eclosion (Fig. 5D). Loss of p132 had no impact on the levels of Retin $\left(\right.$ nina $C^{\Delta 132}$ ) (Fig. 5E). Thus, expression of Retin was strictly dependent on p174.

The reduction in PKC in retin-deficient flies raised the possibility that PKC levels were also reduced in the ninaC mutant. Consistent with the absence of Retin in nina $C^{\Delta 174}$ flies, the concentration of PKC was also diminished in 7-d-old ninaC mutant animals (Fig. 5F, G). As expected, because ninaC flies did not display a PDA (Montell and Rubin, 1988), Rh1 levels were also reduced in $n i-$ $n a C^{\Delta 174}$ flies (Fig. $5 F, G$ ). Thus, NINAC p174 was required for expression of Retin, rhodopsin, and PKC. Similar to what we found in retin ${ }^{1}$ photoreceptor cells, the reduction of Rh1 and PKC in ni$n a C^{\Delta 174}$ flies was age dependent, because the levels of Rh1 and PKC in 1-d-old nina $C^{\Delta 174}$ were indistinguishable from wild type (supplemental Fig. 4A, $B$, available at www.jneurosci.org as supplemental material).

Loss a PKC in retin ${ }^{1}$ was attributable to a requirement for NINAC for stability of INAD

The finding that the concentration of PKC undergoes an agedependent decline in $\operatorname{retin}^{1}$ raises the question as to the molecular
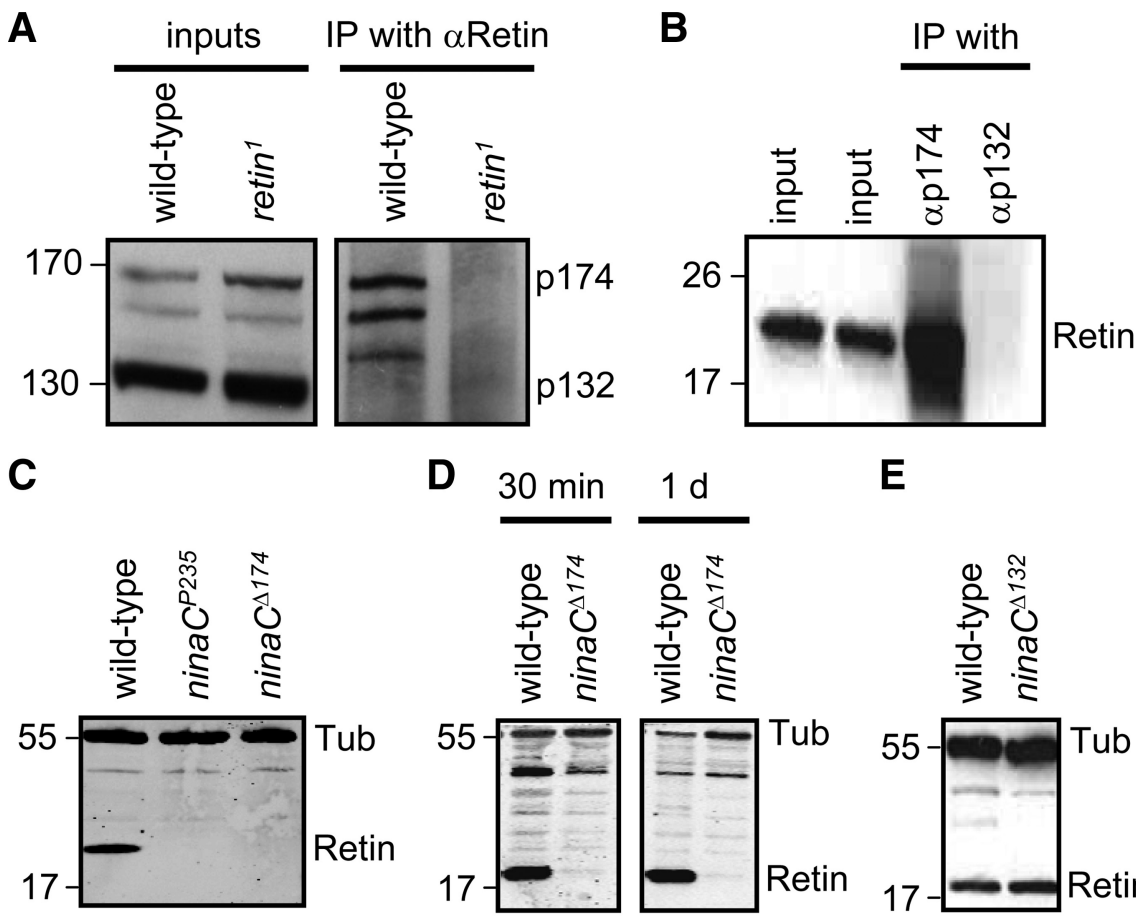

\section{E}

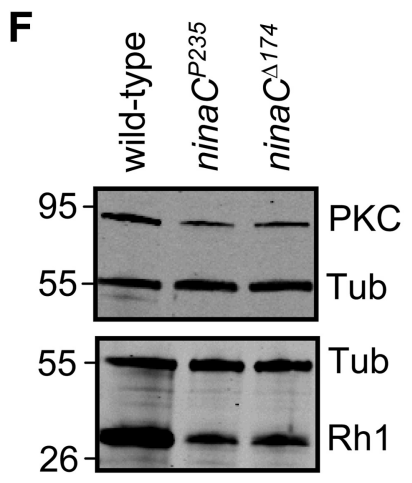

G

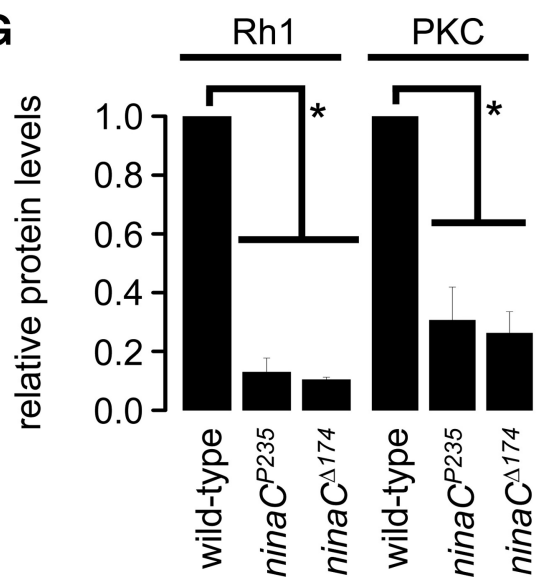

Figure 5. Retin and NINAC p174 interact. $\boldsymbol{A}, \mathrm{p} 174$, but not p132, coimmunoprecipitated with Retin. Retin was immunoprecipitated (IP) with Retin antibodies, and a Western blot was probed with antibodies that recognize both NINAC isoforms. The band immunoprecipitations. $\boldsymbol{B}$, Retin coimmunoprecipitated with p174 but not p132. The p132 or p174 proteins were immunoprecipiwith antibodies specific to each protein, and a Western blot was probed with Retin antibodies. Inputs in $\boldsymbol{A}$ and $\boldsymbol{B}$ represent $5 \%$ of the total. C, Western blot showing the Retin levels in head extracts from 7-d-old flies. Tub, Tubulin. D. Western blots showing the evels of Retin in head extracts from 30-min-old and 1-d-old flies. $\boldsymbol{E}$, Western blot showing the levels of Retin in head extracts. $\boldsymbol{F}$ Western blots showing the levels of PKC and Rh1 in head extracts from 7-d-old flies. Tubulin (Tub) was used as a loading control in all panels. G, Quantification of the relative PKC and Rh1 levels from Western blots. Error bars indicate SEM. ${ }^{*} p<0.05$, ANOVA, $n \geq 3$.

basis for this impairment. NINAC p174 forms a complex with Retin and is dependent on this interaction for protein stability. NINAC p174 also binds the PDZ domain containing protein INAD (Wes et al., 1999), and PKC depends on binding to this scaffold protein for stability (Tsunoda et al., 1997). Therefore, we tested whether the level of PKC might decline in $\mathrm{retin}^{1}$ because of a requirement for NINAC p174 for maintaining normal levels of INAD.

We found that expression of the INAD protein decreased in retin $^{1}$, and this was attributable to a requirement for NINAC p174 for stability of INAD. In 7-d-old flies lacking Retin, there was an $\sim 50 \%$ reduction in the levels of INAD (Fig. $6 A, B$ ) (ANOVA, $p<$ $0.05, n \geq 3)$. This dependence of INAD on Retin was not reciprocal because the amount of Retin in inaD null flies (inaD ${ }^{1}$ ) 
A

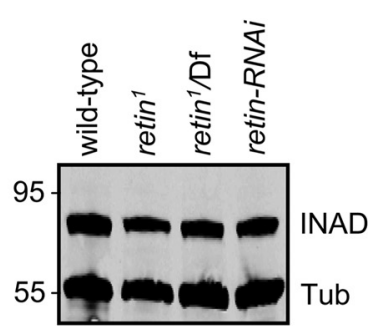

B

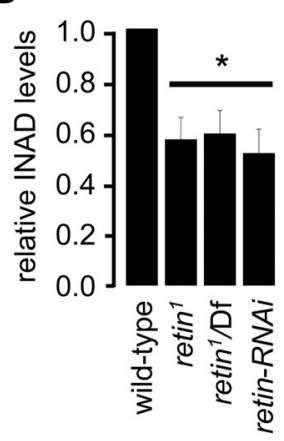

C

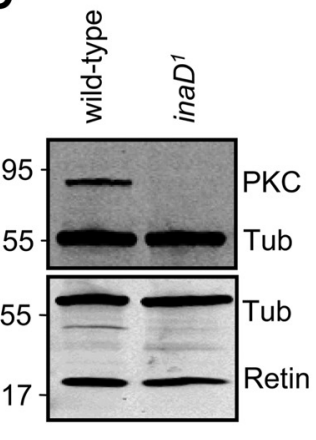

D

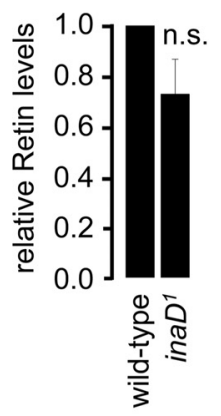

E

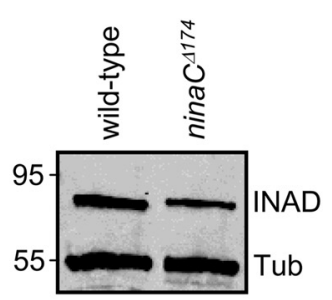

I

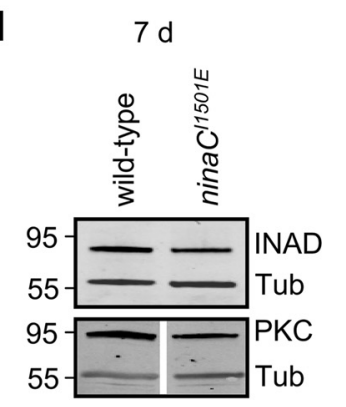

F

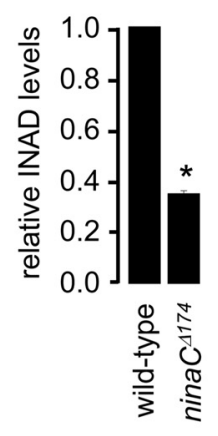

J

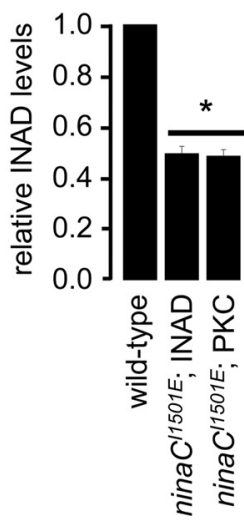

G

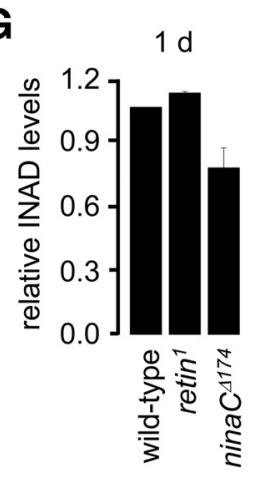

K
H

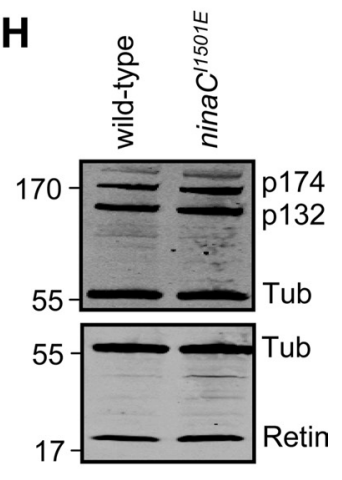

L $\quad 1$ d
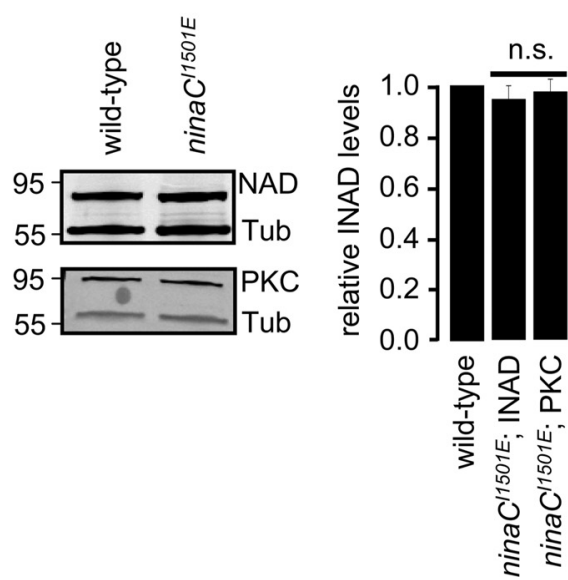

Figure 6. Stability of INAD and PKC depends on NINAC p174. A, Western blot showing the relative levels of INAD in head extracts from 7-d-old wild-type and flies that do not express Retin. The blot was probed with both INAD and Tubulin (Tub) antibodies. B, Quantification of the relative protein levels from blots such as in $A .{ }^{*} p<0.05$, ANOVA, $n \geq 3$. C, Western blots of head extracts from 7-d-old wild-type and inaD flies probed with PKC, Retin, and Tubulin antibodies. D, Quantification of the relative Retin levels from analyses represented in C. n.s., Not significant. $n \geq 3$. E, Western blot of 7-d-old wild-type and nina $C^{\Delta 174}$ head extracts probed with INAD and Tubulin antibodies. $\boldsymbol{F}$, Quantification of the relative INAD levels from $\boldsymbol{E}$ and duplicate blots. $p<0.05$, Student's $t$ test, $n \geq 3$. G, Quantification of the relative INAD levels from Western blots using 1-d-old retin ${ }^{1}$ and nina ${ }^{\Delta 174}$ flies. $\boldsymbol{H}$, Western blots of head extracts from 7-d-old wild-type and nina $C^{17501 E}$ flies probed with NINAC (to common region of 132 and p174), Retin, and Tubulin antibodies. I, Western blots showing the levels of INAD and PKC in head extracts from 7-d-old nina ${ }^{1501 E}$ flies. J, Quantification of the relative INAD and PKC levels from the Western blots such as in $I . K$, Western blots showing the levels of INAD and PKC in head extracts from 1-d-old nina $C^{11501 E}$ flies. $L$, Quantification of the relative INAD and PKC levels from the Western blots such as in $K .{ }^{*} p<0.05$, ANOVA, $n \geq 3$. All error bars indicate \pm SEM.

(Tsunoda et al., 1997; Wes et al., 1999) was not significantly different from wild type (Fig. 6C,D) (Student's $t$ test, $n \geq 3$ ). We found that nina $C^{\Delta 174}$ flies also showed a significant decrease in INAD (Fig. 6E, $F$ ) (Student's $t$ test, $n \geq 3$ ). As was the case for $\mathrm{PKC}$, the reductions in the concentration of INAD in the retin ${ }^{1}$ and ninaC $C^{\Delta 174}$ flies were age dependent, because the levels of INAD in 1-d-old mutant flies were either unchanged or reduced to a smaller extent than in 7-d-old retin ${ }^{1}$ and ninaC ${ }^{\Delta 174}$ (Fig. 6G) (ANOVA, $p>0.05, n \geq 3$ ).

Because both NINAC and Retin mutually affect the concentration of the other protein, we could not distinguish whether the reductions of INAD in retin ${ }^{1}$ or nina $C^{\Delta 174}$ flies were attributable to a requirement for one or the other protein. Therefore, we examined the protein level of INAD in flies that expressed an NINAC variant with a single amino acid change of the C-terminal residue (nina $C^{I 1501 E}$ ), which prevented binding to INAD (Wes et al., 1999). The nina $C^{11501 E}$ flies expressed wild-type amounts of NINAC and Retin (Fig. $6 H$ ). In contrast, the concentrations of INAD and PKC were reduced $\sim 50 \%$ in nina $C^{I 1501 E}$ flies (Fig. $6 I, J)$. These decreases of INAD and PKC in the nina $C^{I 1501 E}$ flies were also age dependent because the levels of INAD and PKC in 1 -d-old ninaC $C^{I 1501 E}$ flies were indistinguishable from the wild- 
type value (Fig. $6 K, L$ ) (ANOVA, $p>0.05, n \geq 3$ ). Although the ina $D^{1}$ null mutation caused instability of TRP, PLC, and PKC, the twofold reduction in INAD in retin $^{l}$ (Fig. 6B) did not result in a significant decline in PLC or TRP (Fig. $4 A$ ) (supplemental Fig. $5 A, B$, available at www.jneurosci.org as supplemental material). Thus, the stabilities of PLC and TRP were less sensitive to the concentration of INAD. Nevertheless, our results indicate that the decrease in INAD results from loss of the NINAC/INAD interaction rather than nonspecific effects on the concentration of either Retin or NINAC, because the both Retin and NINAC p174 were expressed at normal levels in ninaC ${ }^{I 1501 E}$ flies.

\section{Discussion}

In this study, we describe the identification of Retin, a protein required for termination of the photoresponse. Unlike other proteins that function in termination, the retin phenotype was age dependent. Slow termination leads to increased endocytosis and degradation of the major rhodopsin, Rh1, which serves as a negative feedback mechanism to attenuate the visual response (Han et al., 2007). Consistent with a defect in termination, the agedependent impairment in the photoresponse in retin ${ }^{1}$ was associated with greater endocytosis of Rh1 and an age-dependent reduction in the concentration of Rh1.

A central question concerns the basis for the age-dependent decrease in the termination rate in retin-deficient flies. Retin has been reported to function in macrophages through a pathway that involves the ryanodine receptor, a store-operated channel, Orai, and the interacting protein STIM1 (Cuttell et al., 2008), which is present in the endoplasmic reticulum (ER) and senses changes in $\mathrm{ER} \mathrm{Ca}^{2+}$. However, $\mathrm{Ca}^{2+}$ release from the $\mathrm{ER}$, the ryanodine receptor, and the $\mathrm{IP}_{3}$ receptor do not appear to function in Drosophila visual transduction (Acharya et al., 1997; Raghu et al., 2000; Sullivan et al., 2000). Furthermore, knockdown of stim1 RNA using a photoreceptor cell GAL4 in combination with UAS-stim1-RNAi transgene had no effect on phototransduction, the concentration of Retin, or other proteins reduced in retin ${ }^{1}$ mutant eyes (data not shown). The decrease in termination in retin $^{1}$ mutant flies was not directly attributable to loss of Retin, because the Retin protein was absent in young flies that exhibited normal termination. The retin phenotype also was not a consequence of a reduction in NINAC p174, because both 3 - and 7-d-old retin ${ }^{1}$ flies displayed similarly low levels of p174; however, only the 7 -d-old flies exhibited the slow termination phenotype.

We conclude that the age-dependent termination phenotype in retin $^{1}$ results from a reduction in PKC levels. Consistent with this proposal, the decline in PKC concentration paralleled the appearance of the termination phenotype. In young retin ${ }^{1}$ flies, which displayed normal termination, PKC was not reduced significantly from wild type. However, in older retin ${ }^{1}$ flies, the PKC concentration declined twofold. In further support of the conclusion that the $50 \%$ decrease in PKC is responsible for the termination defect in retin $^{1}$, a similar impairment in termination occurs in heterozygous flies, which are missing a copy of the gene encoding the eye-enriched PKC (N. Wang et al., 2008).

We propose the following mechanism through which Retin affects the concentration of PKC. First, Retin forms a complex with NINAC p174, and this interaction is required for the stability of $\mathrm{p} 174$. Both proteins coimmunoprecipitated from head extracts, and loss of Retin resulted in a lower concentration of p174. The requirement for Retin and NINAC was mutual because Retin was undetectable in flies missing p174. Second, NINAC is required for stabilizing the PDZ-containing scaffold protein INAD.
NINAC and INAD interact (Wes et al., 1999), and we find that a single amino acid mutation that disrupts the INAD binding site in p174 (nina $C^{I 1501 E}$ ) causes a reduction in INAD. Third, PKC binds stoichiometrically to INAD and requires this interaction for stability (Huber et al., 1996; Tsunoda et al., 1997; Xu et al., 1998). As a result, INAD and PKC displayed indistinguishable twofold decreases in protein levels. We found that PKC also declined to a similar extent in flies expressing NINAC ${ }^{\mathrm{I1501E}}$. Because INAD was reduced in nina $C^{1501 E}$ flies, but not Retin or NINAC p174, the instability of PKC was not attributable to nonspecific effects resulting from changes in the concentrations either Retin or p174. Thus, loss of Retin causes a reduction in the level of p174, which in turn affects the concentration of INAD, leading to instability of PKC, which underlies the slower termination.

Despite the defect in termination, retin ${ }^{1}$ flies exhibited only minor effects on retinal morphology. There are multiple examples of mutations that are associated with termination defects that display relatively minor alterations in rhabdomere morphology. These include rac2 (Elsaesser et al., 2010), ninaC (Porter et al., 1992), and stops (T. Wang et al., 2008). Of particular relevance, flies heterozygous for a mutation disrupting the eyeenriched PKC (inaC $C^{P 209} /+$ flies), which exhibit a termination phenotype similar to retin $^{1}$ (N. Wang et al., 2008), do not undergo retinal degeneration.

Finally, both Retin and myosins with fused N-terminal protein kinase domains are found in other organisms, including humans. Protein kinase/myosins (myosin IIIs) and Retin are both expressed in the mammalian retina (Dosé and Burnside, 2000; Mecklenburg, 2007). This raises the possibility that Retin and myosins related to NINAC may form a complex in mammalian photoreceptor cells and are required for signaling.

\section{References}

Acharya JK, Jalink K, Hardy RW, Hartenstein V, Zuker CS (1997) InsP receptor essential for growth and differentiation but not for vision in Drosophila. Neuron 18:881-887.

Bloomquist BT, Shortridge RD, Schneuwly S, Perdew M, Montell C, Steller H, Rubin G, Pak WL (1988) Isolation of a putative phospholipase C gene of Drosophila, norpA, and its role in phototransduction. Cell 54:723-733.

Chevesich J, Kreuz AJ, Montell C (1997) Requirement for the PDZ domain protein, INAD, for localization of the TRP store-operated channel to a signaling complex. Neuron 18:95-105.

Cook B, Bar-Yaacov M, Cohen Ben-Ami H, Goldstein RE, Paroush Z, Selinger Z, Minke B (2000) Phospholipase C and termination of G-protein mediated signalling in vivo. Nat Cell Biol 2:296-301.

Cuttell L, Vaughan A, Silva E, Escaron CJ, Lavine M, Van Goethem E, Eid JP, Quirin M, Franc NC (2008) Undertaker, a Drosophila Junctophilin, links Draper-mediated phagocytosis and calcium homeostasis. Cell 135:524-534.

Dolph PJ, Ranganathan R, Colley NJ, Hardy RW, Socolich M, Zuker CS (1993) Arrestin function in inactivation of G protein-coupled receptor rhodopsin in vivo. Science 260:1910-1916.

Dosé AC, Burnside B (2000) Cloning and chromosomal localization of a human class III myosin. Genomics 67:333-342.

Elsaesser R, Kalra D, Li R, Montell C (2010) Light-induced translocation of Drosophila visual Arrestin2 depends on Rac2. Proc Natl Acad Sci U S A 107:4740-4745.

Franceschini N, Kirschfeld K, Minke B (1981) Fluorescence of photoreceptor cells observed in vivo. Science 213:1264-1267.

Fu Y, Yau KW (2007) Phototransduction in mouse rods and cones. Pflugers Arch 454:805-819.

Gong WJ, Golic KG (2003) Ends-out, or replacement, gene targeting in Drosophila. Proc Natl Acad Sci U S A 100:2556-2561.

Han J, Reddig K, Li HS (2007) Prolonged $\mathrm{G}_{\mathrm{q}}$ activity triggers fly rhodopsin endocytosis and degradation, and reduces photoreceptor sensitivity. EMBO J 26:4966-4973. 
Hankins MW, Peirson SN, Foster RG (2008) Melanopsin: an exciting photopigment. Trends Neurosci 31:27-36.

Hardie RC, Minke B (1992) The trp gene is essential for a light-activated $\mathrm{Ca}^{2+}$ channel in Drosophila photoreceptors. Neuron 8:643-651.

Huber A, Sander P, Gobert A, Bähner M, Hermann R, Paulsen R (1996) The transient receptor potential protein (Trp), a putative store-operated $\mathrm{Ca}^{2+}$ channel essential for phosphoinositide-mediated photoreception, forms a signaling complex with NorpA, InaC and InaD. EMBO J 15:7036-7045.

Lee SJ, Montell C (2001) Regulation of the rhodopsin protein phosphatase, RDGC, through interaction with calmodulin. Neuron 32:1097-1106.

Li HS, Montell C (2000) TRP and the PDZ protein, INAD, form the core complex required for retention of the signalplex in Drosophila photoreceptor cells. J Cell Biol 150:1411-1422.

Liu CH, Satoh AK, Postma M, Huang J, Ready DF, Hardie RC (2008) $\mathrm{Ca}^{2+}$ dependent metarhodopsin inactivation mediated by calmodulin and NINAC myosin III. Neuron 59:778-789.

Mecklenburg KL (2007) Drosophila retinophilin contains MORN repeats and is conserved in humans. Mol Genet Genomics 277:481-489.

Montell C, Rubin GM (1988) The Drosophila ninaC locus encodes two photoreceptor cell specific proteins with domains homologous to protein kinases and the myosin heavy chain head. Cell 52:757-772.

Montell C, Rubin GM (1989) Molecular characterization of the Drosophila trp locus: a putative integral membrane protein required for phototransduction. Neuron 2:1313-1323.

Pak WL (1979) Study of photoreceptor function using Drosophila mutants. In: Genetic approaches to the nervous system (Breakfield XO, ed), pp 67-99. New York: Elsevier.

Popescu DC, Ham AJ, Shieh BH (2006) Scaffolding protein INAD regulates deactivation of vision by promoting phosphorylation of Transient Receptor Potential by eye protein kinase C in Drosophila. J Neurosci 26:8570-8577.

Porter JA, Montell C (1993) Distinct roles of the Drosophila ninaC kinase and myosin domains revealed by systematic mutagenesis. J Cell Biol 122:601-612.

Porter JA, Hicks JL, Williams DS, Montell C (1992) Differential localizations of and requirements for the two Drosophila ninaC kinase/myosins in photoreceptor cells. J Cell Biol 116:683-693.

Porter JA, Yu M, Doberstein SK, Pollard TD, Montell C (1993) Dependence of calmodulin localization in the retina on the ninaC unconventional myosin. Science 262:1038-1042.

Porter JA, Minke B, Montell C (1995) Calmodulin binding to Drosophila NinaC required for termination of phototransduction. EMBO J 14:44504459.

Raghu P, Colley NJ, Webel R, James T, Hasan G, Danin M, Selinger Z, Hardie RC (2000) Normal phototransduction in Drosophila photoreceptors lacking an $\mathrm{InsP}_{3}$ receptor gene. Mol Cell Neurosci 15:429-445.
Scott K, Sun Y, Beckingham K, Zuker CS (1997) Calmodulin regulation of Drosophila light-activated channels and receptor function mediates termination of the light response in vivo. Cell 91:375-383.

Shieh BH, Niemeyer B (1995) A novel protein encoded by the InaD gene regulates recovery of visual transduction in Drosophila. Neuron 14:201-210.

Smith DP, Ranganathan R, Hardy RW, Marx J, Tsuchida T, Zuker CS (1991) Photoreceptor deactivation and retinal degeneration mediated by a photoreceptor-specific protein kinase C. Science 254:1478-1484.

Sullivan KM, Scott K, Zuker CS, Rubin GM (2000) The ryanodine receptor is essential for larval development in Drosophila melanogaster. Proc Natl Acad Sci U S A 97:5942-5947.

Takeshima H, Komazaki S, Nishi M, Ino M, Kangawa K (2000) Junctophilins: a novel family of junctional membrane complex proteins. Mol Cell 6:11-22.

Tsunoda S, Sierralta J, Sun Y, Bodner R, Suzuki E, Becker A, Socolich M, Zuker CS (1997) A multivalent PDZ-domain protein assembles signalling complexes in a G-protein-coupled cascade. Nature 388:243-249.

Vinós J, Jalink K, Hardy RW, Britt SG, Zuker CS (1997) A G proteincoupled receptor phosphatase required for rhodopsin function. Science 277:687-690.

Walsh T, Walsh V, Vreugde S, Hertzano R, Shahin H, Haika S, Lee MK, Kanaan M, King MC, Avraham KB (2002) From flies' eyes to our ears: mutations in a human class III myosin cause progressive nonsyndromic hearing loss DFNB30. Proc Natl Acad Sci U S A 99:7518-7523.

Wang N, Leung HT, Pak WL, Carl YT, Wadzinski BE, Shieh BH (2008) Role of protein phosphatase $2 \mathrm{~A}$ in regulating the visual signaling in Drosophila. J Neurosci 28:1444-1451.

Wang T, Montell C (2007) Phototransduction and retinal degeneration in Drosophila. Pflugers Arch 454:821-847.

Wang T, Xu H, Oberwinkler J, Gu Y, Hardie RC, Montell C (2005) Light activation, adaptation, and cell survival functions of the $\mathrm{Na}^{+} / \mathrm{Ca}^{2+}$ exchanger CalX. Neuron 45:367-378.

Wang T, Wang X, Xie Q, Montell C (2008) The SOCS box protein STOPS Is required for phototransduction through Its effects on phospholipase C. Neuron 57:56-68.

Wes PD, Xu XZ, Li HS, Chien F, Doberstein SK, Montell C (1999) Termination of phototransduction requires binding of the NINAC myosin III and the PDZ protein INAD. Nat Neurosci 2:447-453.

Xu H, Lee SJ, Suzuki E, Dugan KD, Stoddard A, Li HS, Chodosh LA, Montell C (2004) A lysosomal tetraspanin associated with retinal degeneration identified via a genome-wide screen. EMBO J 23:811-822.

Xu XZ, Choudhury A, Li X, Montell C (1998) Coordination of an array of signaling proteins through homo- and heteromeric interactions between PDZ domains and target proteins. J Cell Biol 142:545-555. 